\title{
Real-time Visual Landmark Recognition in Multi-view Image Collections
}

\author{
Kwisha Hitesh Gohil \\ Student \\ Department of Information Technology \\ Chandubhai S. Patel Institute of Technology \\ (CSPIT) \\ Charotar University of Science and Technology
}

(CHARUSAT)

\author{
Sonal Pravinbhai Rami \\ Assistant Professor \\ Department of Information Technology \\ Chandubhai S. Patel Institute of Technology \\ (CSPIT) \\ Charotar University of Science and Technology
}

(CHARUSAT)

\begin{abstract}
Research and advancement in the Convolution Neural Network have been capable of solving many computer vision problems with higher accuracy than humans at some time. This paper, presents CNN along with its various layers for easy understanding. CNN algorithm has been used here for the landmark recognition problem. In the 3D Visual Phrasing method, SfM has been used to reconstruct a 2D image of a landmark to its 3D image for better classification. To solve the problem of landmark recognition, various approaches have been put forward. Each approach mentioned in the paper is an enhancement of the previously mentioned approach to obtain greater accuracy in landmark recognition.
\end{abstract}

\section{Keywords}

3D Visual Phrase, CleverHans, Convolution Neural Network, Deep learning, Keras, Landmark Recognition, Machine learning, Object detection, Pre-trained models, TensorFlow

\section{INTRODUCTION}

Humans have proved to be one of the most intelligent species on the planet. But, with the advent of growing technological innovations, computers are not far behind. Humans have always been keen to know in which place their friend clicked that intriguing photo that has been posted on the social media page. The first attempt is always to figure it out by themselves before asking. In order to do so, cues from the images are picked out. These cues include architectural styles, landmarks, road signs, driving styles and other information that is available in the photo. Imagine a computer doing the same task for humans. It will save a lot of time.

\section{BACKGROUND THEORY}

Computer Vision is the technology of making computers identify and process the images just like the humans do. The aim of computer vision is to extract all possible information and analyze it using various parameters to obtain useful information. It can use either a single image or a stream of images depending on its purpose. The technology giants like Microsoft and Google have made significant developments in this field. It gave rise to the Convolution Neural Network (CNN) which uses less preprocessing than other techniques for image classification. Classification is a technique in which the data is processed with the purpose of categorizing them in multiple target classes. Numerous models using the techniques of SVM, Linear Classifier, Bayes' Classifier and Neural networks have been proposed for image processing. Convolution Neural Network has been used here.

\section{CONVOLUTION NEURAL NETWORK}

Convolution Neural Network is a classification technique. It is a type of feed-forward Artificial Neural Network. It consists of weights that are learnable along with certain bias. For understanding $\mathrm{CNN}$, an example of image is taken as shown below in the Fig. 1. The numbers in the figure represent the pixel values of the image.

\begin{tabular}{|c|c|c|c|c|}
\hline 67 & 96 & 42 & 77 & 84 \\
\hline 94 & 120 & 112 & 64 & 62 \\
\hline 100 & 76 & 96 & 88 & 54 \\
\hline 72 & 43 & 68 & 97 & 117 \\
\hline 85 & 78 & 80 & 111 & 90 \\
\hline
\end{tabular}

Fig 1: $5 * 5$ Image

\subsection{Convolution Layer}

It takes the features from the images and moves the filters, whose size is decided previously, throughout the image while continuously multiplying the image pixel value with the filter pixel value and dividing the result with the total number of pixels. Appropriate user defined filters/features are selected from the image.

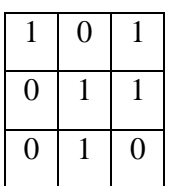

Fig 2: $3 * 3$ Filter 1

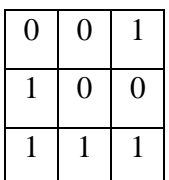

Fig 3: $3 * 3$ Filter 2

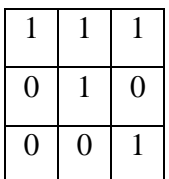

Fig 4: $3 * 3$ Filter 3

Take one filter and scan it through the entire image. Select 3 while multiplying each image pixel with feature pixel. 


\begin{tabular}{|c|c|c|c|c|}
\hline 67 & 96 & 42 & 77 & 84 \\
\hline 94 & 120 & 112 & 64 & 62 \\
\hline 100 & 76 & 96 & 88 & 54 \\
\hline 72 & 43 & 68 & 97 & 117 \\
\hline 85 & 78 & 80 & 111 & 90 \\
\hline
\end{tabular}

Fig 5: Original Image

\begin{tabular}{|c|c|c|c|c|c|c|}
\hline 1 & 0 & 1 & & 417 & 445 & 340 \\
\cline { 1 - 2 } \cline { 5 - 6 } & 1 & 1 & 421 & 436 & 413 \\
\hline 0 & 1 & 0 & & 385 & 409 & 475 \\
\cline { 1 - 1 } & &
\end{tabular}

Filter 1Output Image1

Fig. 6. Passing of the Filter 1 through the Original Image

\begin{tabular}{|c|c|c|c|c|c|c|}
\hline 0 & 0 & 1 & \multirow{2}{*}{} & 408 & 457 & 434 \\
\hline 1 & 0 & 0 & 395 & 348 & 440 \\
\hline 1 & 1 & 1 & & 411 & 400 & 403 \\
\cline { 1 - 2 } & &
\end{tabular}

Filter 2Output Image 2

Fig. 7. Passing of the Filter 2 through the Original Image

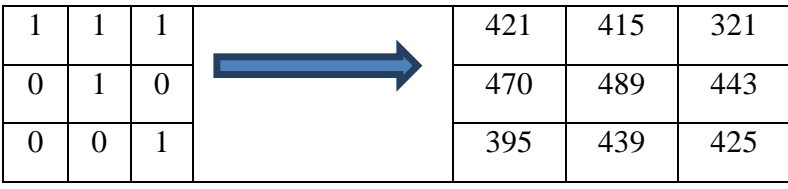

Filter 3 Output Image 3

Fig. 8. Passing of the Filter 3 through the Original Image

LRF-Local Receptive Field

Output image value $=\mathrm{LRF}^{*}$ Filter

(dot product of LRF and Filter)

Size of filter $=3 \times 3$

Size of input $=5 \times 5$

Stride $\quad=1 \times 1($ move 1 cell $)$

Padding $=0 \mathrm{x} 0$ (No padding)

\subsubsection{Formula}

Output Size $=\{$ Input Size - Filter Size $+(2 \times$ Padding $)\}$ $\times$ Stride +1

Calculating Output size from the above equation,

Output size $=(I-F+2 P) * S+1$

Output size $=(5-3) * 1+1$

Output size $=3 \times 3$

\subsection{Pooling Layer}

Pooling layer is used to shrink the image by taking a specific window size. There can be multiple pooling layers in a CNN.

Taking the maximum values of the result obtained from the above layers, the following matrix is obtained by keeping the parameters as follows:

Pool size $=2 \times 2$

Pool type $=$ Max-pool

Stride $=2$
Padding $=1$

\begin{tabular}{|l|l|}
\hline 445 & 445 \\
\hline 409 & 475 \\
\hline
\end{tabular}

Fig. 9. Output After applying Max Pooling on Output Image 1

\subsection{Relu Layer (Rectified Linear Unit)}

$\mathrm{ReLu}$ is an activation Layer which is used to remove the negative values from the obtained matrix of the previous layer. The following equation is used at the ReLu Layer.

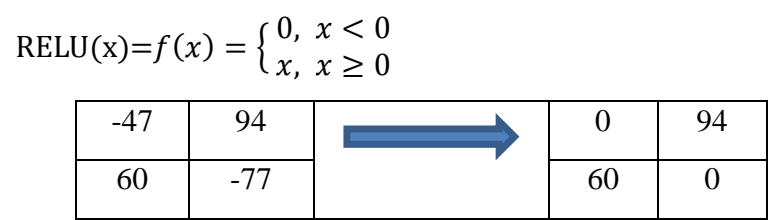

Fig. 10. Output After Passing through ReLu Layer

\subsection{Fully Connected Layer}

This layer converts the set of values obtained from all the previous layers to the type of values which have been given in the final output layer so that a precise comparison can be made between the two.

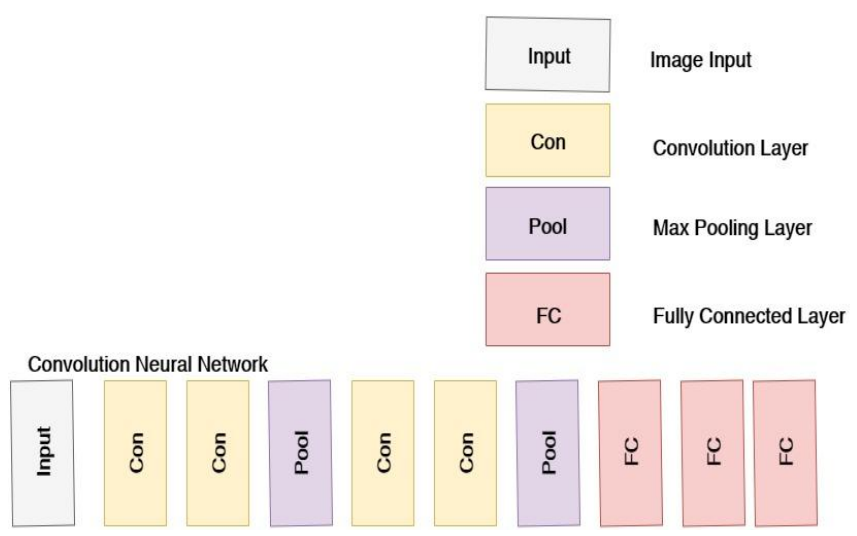

Fig. 11. Convolution Neural Network

\section{IMAGE DETECTION AND ITS VARIOUS APPROACHES}

A large number of approaches have been devised for image classification using various techniques.

This paper [1], uses Yahoo Flickr Creative Commons $100 \mathrm{M}$ (YFCC100M) dataset, consisting of a large number of images from Flickr. It also uses the Hashtag Context Feature of the Instagram along with the Visual Context Feature, which takes help of the surrounding images at the same location. It uses the Bayes' Rule for solving of the problem which takes into consideration the co-ordinates of the geotagged image (the latitude and longitude of the image). The use of longitude and latitude helps to know the exact location of the image with the help of American Community Service (ACS). A tedious task when using this approach is to manually verify the discarded images so that they do not contain the classes that are of interest. However, this approach fails at obtaining accuracy for the images of car brands which are not location specific. This study, considers RL10 as optimal. (Here RL10 refers to the number of replicas of the radius learning layer).

This paper [2], uses Pre-trained models such as GoogleNet and VGG16 Net for the geolocating of their dataset. They selected 4,672,382 images from the MediaEval challenge and processed them using the Vanilla CNN technique. However, the testing 
dataset was confined to a similar number of images from only 5 countries from all over the world making the experiment a success only when the geo-locating was confined to a specific area. The best performance was obtained on visual data within $100 \mathrm{~km}$ only. $100 \mathrm{~km}$ can roughly be considered as the radius of a small country. This makes the approach infeasible for using on the entire map of the earth. If working on a small Dataset, this approach can be considered as useful.

An outstanding work done by google [3], is developing its PlaNet Model. By removing the parameters of latitude and longitude as these parameters would get elongated near the poles and replacing them by $\mathrm{S} 2$ cells from the Google's open Source S2 geometry library, which increases the efficiency of the Advanced CNN model. For an even deeper analysis to the problem, LSTM architectures [4] have been used. This model was then tested on the INRIA Holiday dataset. This model fails at correctly determining the location of the images in the rural areas as the pictures taken in the rural areas will be less than that taken in the urban areas and the probability of the images in the rural areas having a landmark to detect via this model will be very low. When google compared PlaNet with humans in the Geoguessr challenge, it was found that both of these could only identify images at the country or the state level and had a lower success rate when the images reached below the street or the city level. Proving that this task was much more difficult than the Flickr and Im2GPS datasets.

\section{EXPERIMENTAL SETUP}

The goal is to use a pretrained model in order to recognize the landmarks for processing the image. Maximum pooling is taken into consideration while doing it so that maximum accuracy is obtained in the model. The co-ordinates of the image (latitude and longitude) have been ignored as these parameters get elongated at the poles. The bag-of-words framework is not used as it has a few limitations. When BoW framework is used, it extracts features from irrelevant objects like road signs, faces, animals, etc. Apart from this, it ignores the geometric relationship between the database images.

The structure-from-motion (SfM) $[5,6,7]$ is used to reconstruct 3D Models from a set $\mathcal{J}$ of $2 \mathrm{D}$ images by maintaining the geometric relationship between the images and removing the irrelevant noisy objects.

The 3D model is covered by a point cloud composed of a set $\mathcal{L}$ of $3 \mathrm{D}$ points. For each $3 \mathrm{D}$ point $\ell$ belongs to $\mathcal{L}$ is selected if it corresponds to a point in the set $\mathcal{J}$ of the reconstructed 3D Models. The points are selected based on the point popularity which means selecting a point having maximum occurrence. The selected points should be able to cover all the angles of the specific landmark. These points should collectively make a model of the entire landmark.

In the next step, several points are combined in a group such that the visual phrases are derived from the facets on the surface of the 3D landmark Model. Each of the facet should have sound visibility and repeatability in unseen images. The surface of the Model is approximated using a number of Delaunay Triangles.

The $3 \mathrm{D}$ visual phrase having three vertex points is characterized by two perspectives. Visual Appearance provides relaxed criteria to recall true positives and geometric structures serves as constraints to eliminate false positives and boost the precision [8].

\subsection{Dataset Description}

Lanmark3d has been taken as the database for landmark recognition using 3D models.

- 25 landmarks, each with a 3D model (point cloud) which are to be reconstructed using structure-frommotion (SfM) [8].

- $\quad 45,180$ database images with approximately $1.4 \mathrm{~K} \sim 2 \mathrm{~K}$ per landmark which have been registered to the $3 \mathrm{D}$ models [8].

- 10,000 positive test images for evaluation (400 per landmark) [8].

- $\quad$ 2.7 million 3D points in the 3D models $(29 \mathrm{~K} \sim 223 \mathrm{~K}$ per landmark) [8].

\section{IMPLEMENTATION USING VARIOUS APPROACHES AND THEIR OUTCOMES}

\subsection{Basic CNN}

Using the Basic CNN model, input all the images and set their dimensions as $224 * 224$. As there are 25 Landmarks, the number of classes will be 25 . The pixel values are rescaled from interval $[0,255]$ to the interval $[0,1]$. As a result, 4158 images belonging to the 25 classes have been found. It will have 3 convolution layers, a ReLu activation Layer followed by multiple max pooling layers. A target of 100 epochs is set with 40805 training images and 8856 testing images. With the facility of Early Stopping of the Keras library the execution is stopped when the quality of output stops improving. As a result, the execution stopped after reaching only 15 epochs and the accuracy obtained from this model was merely $60 \%$. This approach was found to be overfitting the model. A graph of Accuracy v/s Epochs is shown in the Fig. 12. which follows.

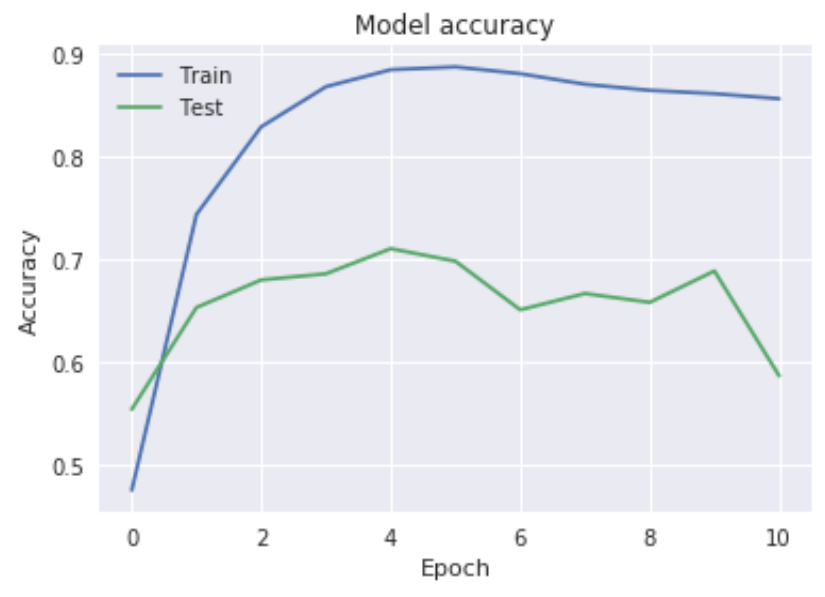

Fig. 12. Accuracy v/s Epoch graph for Basic CNN Approach

\subsection{Basic CNN with Data Augmentation}

In this approach, the dimensions of the image and their rescaling parameters remain the same. However, as the data augmentation approach is used here, it is required to find all possible views of the landmark. A specific shear range and zoom range of 0.2 is taken which cuts the 3D Model so that the images having half of the landmark as well as the zoomed in images of the landmark are also considered in the final output set. The number of images found here for the 25 classes are the same as that found in the Basic CNN model i.e. 4158 images. To show an example of this transformations, the Python Imaging Library is used which can store large number of images in multiple formats. To obtain a 
powerful N-dimensional array, the facility of NumPy library of python is used with the specific dimensions of the array as required when necessary. An outcome is obtained as the Multiple angle view of a single landmark. Again, the Early Stopping functionality of Keras library is used. However, this time the accuracy increases from $60 \%$ to $70 \%$ because of the data augmentation. By this, it is known that this approach is better than the basic CNN model.

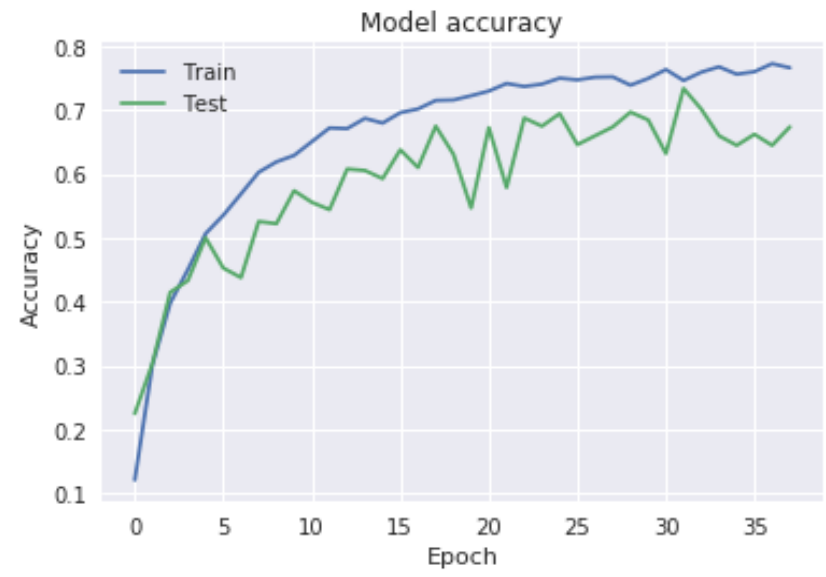

Fig. 13. Accuracy v/s Epoch graph for Basic CNN with Data Augmentation Approach

\subsection{VGG with Multi-layer Perceptron}

In this model also, in the initial stage, the image dimension size and the rescaling parameters remain the same. Here, 4158 images are found belonging to 25 classes such that each landmark has a class of itself. A pretrained VGG16 Model is used in this approach and taking weights of the ImageNet [9]. The to_categorical facility of Keras is used to convert these images into binary class matrix. Alongside, a bottleneck is provided to these images and some images are removed which are not needed. A ReLu activation function is applied such that the number of training images are 40800 and test images are 8832. A multi-layer perceptron is also used in this model. Here, the accuracy obtained is $85 \%$ after just 10 seconds and this approach proved to be even better than basic CNN with data augmentation approach as it consists a bottleneck feature.

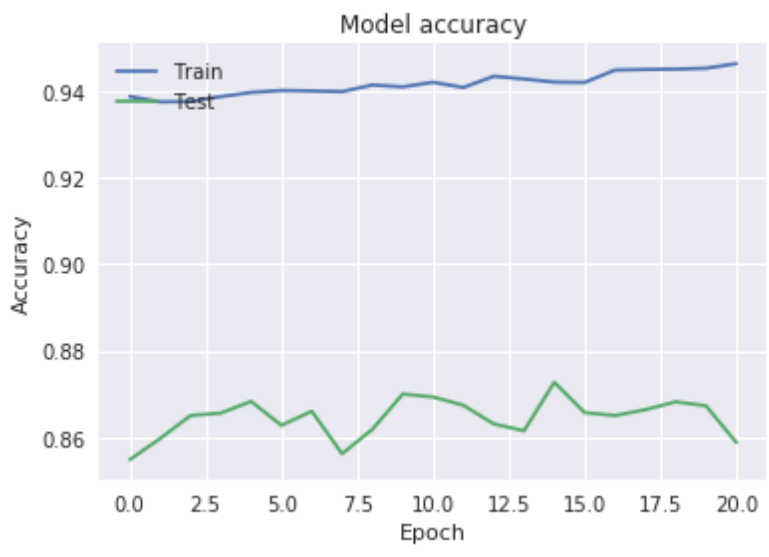

Fig. 14. Accuracy v/s Epoch graph for VGG16 with Multilayer Perceptron Approach

\subsection{Fine-tuning VGG16}

The initial steps of this approach are similar to the ones in VGG16 with Multi-layer perceptron approach. This approach is similar till the ImageNet [9] weights are taken for this pre- trained model. Now, to fine tune the model according to requirement, a classifier model is needed to put above the convolution model. A fully trained classifier is needed, to place on top of the convolution base. The first 15 layers are set as nontrainable as only a few layers are needed for training. For optimization, the optimizer from the Keras library is used, the default SGD (Stochastic gradient descent) optimizer. This optimizer includes support for momentum and learning rate decay. An accuracy of $94 \%$ is obtained using this model after fine-tuning the previous model and hence, this approach works better.

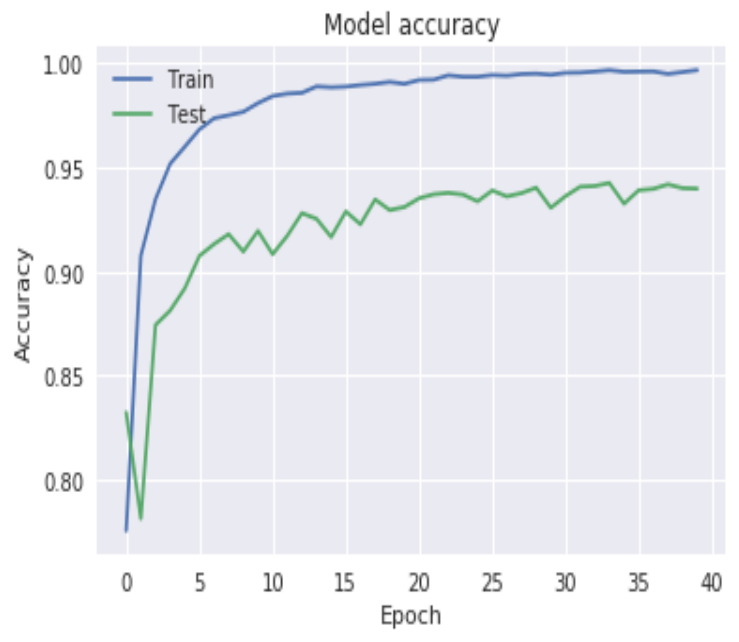

Fig. 15. Accuracy v/s Epoch graph for Fine-tuning VGG16 Approach

\subsection{Adversarial examples with FGSM}

FGSM (Fast Gradient Sign Method) is used to obtain the local minima of a graph by using the loss function. The equation of loss function [10] used is as follows:

$$
L(x, y, a, b)=(y-(a x-b))^{2}
$$

Where $x$ is the original image, $y$ is the class of $x . a$ and $b$ are parameters of the image. The gradient is computed first by using this loss function so that it is known in which direction better results of the input image are obtained. While doing the calculations, only the sign of the slope is necessary to check if an increase in the pixel value or decrease in the pixel value gives a better result.

In this approach, the classes that are supposed to be used for classification are manually input. Then the same steps are performed as for VGG16 with Multi-layer perceptron approach till the resulting images are obtained after using the bottleneck An $\varepsilon$ value of 0.02 is taken which will be considered as the perturbation. The value of $\varepsilon$ has to be small so that it does not go farther away from the loss function surface and the perturbation is almost negligible. This approach gives $100 \%$ confidence with the original image of the landmark.

\subsection{Basic CNN adversarial benchmarks with CleverHans}

CleverHans is a repository as well as a Python library which is used to benchmark the vulnerabilities of machine learning algorithms to adversarial examples. This technique accelerates the graph related computations when using it through TensorFlow, which is another python library. It is a combination of the Basic CNN approach, FGSM approach and CleverHans. Unlike other approaches, this approach generates 8856 images 
belonging to the 25 classes that have been put. The number of images found here is almost double of the previous approaches. While testing this approach on legitimate images, an accuracy of approximately $69.77 \%$ is obtained. Whereas, on testing it on illegitimate images, an accuracy of approximately $20.23 \%$ is obatined. But, as the dataset becomes large in this approach, it is considered that this approach is better when using big data.

\section{COMPARISON OF APPROACHES}

The comparison table mentioned below does not have Adversarial Example with FGSM approach as this approach does not give accuracy, rather it gives us confidence.

Table 1. Comparison of Various approaches

\begin{tabular}{|c|c|c|c|c|c|}
\hline Parameters & Basic CNN & $\begin{array}{c}\text { Basic CNN with } \\
\text { data Augmentation }\end{array}$ & $\begin{array}{c}\text { VGG16 with Multi- } \\
\text { Layer Perceptron }\end{array}$ & $\begin{array}{c}\text { Fine Tuning } \\
\text { VGG16 }\end{array}$ & $\begin{array}{c}\text { *Basic CNN with } \\
\text { CleverHans }\end{array}$ \\
\hline Epochs & 10 & 37 & 20 & 39 & 20 \\
\hline Accuracy (\%) & 60 & 70 & 85 & 94 & 69.77 \\
\hline
\end{tabular}

*The epochs for this approach have to be explicitly defined and the accuracy obtained is for legitimate images.

\section{CONCLUSION}

Various approaches have been used in this paper and the results are provided for each one of them. Each approach mentioned, is an addition to its previous version and better than the previous approach. It can be concluded that while using small datasets which have images of only the classes that have been mentioned, the Adversarial Examples with FGSM approach can be used. However, this is not the real-life situation and the dataset is bound to have a plethora of images that are irrelevant to the classes of choice. At this time, it would be better to go ahead with the CleverHans Approach. A limitation to this problem is that only a small dataset consisting of 25 famous landmarks have been taken in this paper. As an extension to this problem, the landmarks can be identified using PlaNet's geolocating technique and then this approach can be used on a larger dataset to obtain better results.

\section{REFERENCES}

[1] Tang, Kevin, et al. "Improving image classification with location context." Proceedings of the IEEE international conference on computer vision. 2015.

[2] Sy, Angela, and Cynthia Day. "Geo-Locating Images: Where in the world was this picture taken?." (2016).

[3] Weyand, Tobias, Ilya Kostrikov, and James Philbin. "Planet-photo geolocation with convolutional neural networks." European Conference on Computer Vision. Springer, Cham, 2016.
[4] Hochreiter, Sepp, and Jürgen Schmidhuber. "Long shortterm memory." Neural computation 9.8 (1997): 1735-1780.

[5] Snavely, Noah, Steven M. Seitz, and Richard Szeliski. "Photo tourism: exploring photo collections in 3D." ACM transactions on graphics (TOG). Vol. 25. No. 3. ACM, 2006.

[6] Snavely, Noah, Steven M. Seitz, and Richard Szeliski. "Modeling the world from internet photo collections." International journal of computer vision 80.2 (2008): 189-210.

[7] Wu, Changchang, et al. "Multicore bundle adjustment." Computer Vision and Pattern Recognition (CVPR), 2011 IEEE Conference on. IEEE, 2011.

[8] Hao, Qiang, et al. "3d visual phrases for landmark recognition." Computer Vision and Pattern Recognition (CVPR), 2012 IEEE Conference on. IEEE, 2012.

[9] Deng, Jia, et al. "Imagenet: A large-scale hierarchical image database." 2009 IEEE conference on computer vision and pattern recognition. Ieee, 2009.

[10] Goodfellow, Ian J., Jonathon Shlens, and Christian Szegedy. "Explaining and harnessing adversarial examples (2014)." arXiv preprint arXiv:1412.6572. 